\title{
Combining time-resolved optical (TOS), elec- tronic (trARPES) and structural (UED) probes on the class of rare earth tritellurides $\mathrm{RTe}_{3}$
}

\author{
Timm Rohwer ${ }^{1,2,}{ }^{*}$, Alfred Zong ${ }^{1}$, Anshul Kogar ${ }^{1}$, Ya-Qing Bie ${ }^{1}$, Changmin Lee ${ }^{1}$, Edoardo \\ Baldini ${ }^{1}$, Emre Ergecen ${ }^{1}$, Mehmet B. Yilmaz ${ }^{1}$, Byron Freelon ${ }^{1,3}$, Edbert J. Sie ${ }^{1,4}$, Hengyun \\ Zhou $^{1,5}$, Joshua Straquadine $e^{4,6,7}$, Philip Walmsley, ${ }^{4,6,7}$, Pavel E. Dolgirev ${ }^{8}$, Alexander V. \\ Rozhkov $^{8,9}$, Ian R. Fisher ${ }^{4,6,7}$, Pablo Jarillo-Herrero ${ }^{1}$, Boris V. Fine ${ }^{8,10}$, and Nuh Gedik ${ }^{1}$ \\ ${ }^{1}$ Massachusetts Institute of Technology, Department of Physics, Cambridge, MA 02139, USA \\ ${ }^{2}$ Center for Free-Electron Laser Science, DESY, Notkestraße 85, 22607 Hamburg, Germany \\ ${ }^{3}$ University of Louisville, Department of Physics and Astronomy, Louisville, KY 40292, USA \\ ${ }^{4}$ Geballe Laboratory for Advanced Materials, Stanford University, Stanford, CA 94305, USA \\ ${ }^{5}$ Department of Physics, Harvard University, Cambridge, MA 02138, USA \\ ${ }^{6}$ Department of Applied Physics, Stanford University, Stanford, CA 94305, USA \\ ${ }^{7}$ SIMES, SLAC National Accelerator Laboratory, Menlo Park, CA 94025, USA \\ ${ }^{8}$ Skoltech, Skolkovo Innovation Center, 3 Nobel Street, Moscow, 143026, Russia \\ ${ }^{9}$ Institute for Theoretical and Applied Electrodynamics, RAS Moscow, 125412, Russia \\ ${ }^{10}$ ITP, University of Heidelberg, Philosophenweg 12, 69120 Heidelberg, Germany
}

\begin{abstract}
The combination of EUV based time-resolved Angle-ResolvedPhoto-Electron-Spectroscopy (trARPES), Ultrafast-Electron-Diffraction (UED) and Transient-Optical-Spectroscopy (TOS) facilitates a comprehensive study and all-embracing analysis of correlated dynamics, exemplified on the system of Charge-Density-Waves (CDW's) in rare earth tritellurides $\left(\mathrm{RTe}_{3}\right)$.
\end{abstract}

\section{Introduction}

Charge Density Waves (CDW's) are the dominant perturbation in low dimensional crystals. Moreover, CDW phases are most often linked with superconductivity (SC) at their quantum critical points. The quest for the underlying nature of correlated dynamics in condensed-matter, like SC or CDW's has fostered the development and enhancement of the time-resolved probing techniques themselves.

Among these, time-resolved Angle-Resolved-Photo-Electron-Spectroscopy (trARPES) has emerged as a leading technique in identifying dynamic key properties of complex electronic systems [1-3]. Ever since the initial application of High-Harmonic Generation based ARPES on CDW systems [4], we were able to improve the parameters like energy resolution and repetition rate by orders of magnitude. These technical benefits allow new insights by a more precise observation of the dynamics of the electronic structure in energy and momentum.

\footnotetext{
*Corresponding author: timm.rohwer@cfel.de
} 
However, trARPES is only sensitive to correlations of the electronic system. To independently compare the dynamics of the electron and phonon system (lattice) an independent structural probe is required. This has been realized on a lab-scale by a setup for Ultrafast-Electron-Diffraction (UED). Dramatic space charge broadening due to the Coulomb repulsion of the electrons was avoided by a compact design in combination with a high repetition rate. A third technique of Transient Optical Spectroscopy (TOS) further supports the classification of the observed timescales and threshold fluences based on an advanced time-resolution and sensitivity.

\section{Experiment}

The potential of this combined study is presented on the particular system of Charge Density Waves in rare earth tri-tellurides. An unexpected separation of the CDW amplitude and phase recovery is observed and a more complete picture of the non-thermal transition is only provided by the combined analysis of these complementary techniques.

A schematic of the trARPES and UED setup as well as exemplifying data on $\mathrm{LaTe}_{3}$ can be found in Fig. 1. trARPES was performed by an $\mathrm{Yb}: \mathrm{KGW}$ laser at a repetition rate of 250 $\mathrm{kHz}$. The excitation pulses were generated by an Optical-parametric-amplifier (OPA) while for the probe pulses the third harmonic was generated in a nonlinear crystal and again frequency tripled in a hollow core fiber filled with xenon, reaching an energy UV pulses by an off-axis monochromator [5] and send to a Helium-cooled six axis sample manipulation stage. An Angle-Resolved Time-of-Flight Detector allowed the detection of a full 3D spectrum $\left(\mathrm{E}, \mathrm{k}_{\mathrm{x}}\right.$ and $\mathrm{k}_{\mathrm{y}}$ ) with an opening angle of $30^{\circ}$ at a selected sample tilt. The energy and time resolution was determined to $16 \mathrm{meV}$ and $230 \mathrm{fs}$, respectively.

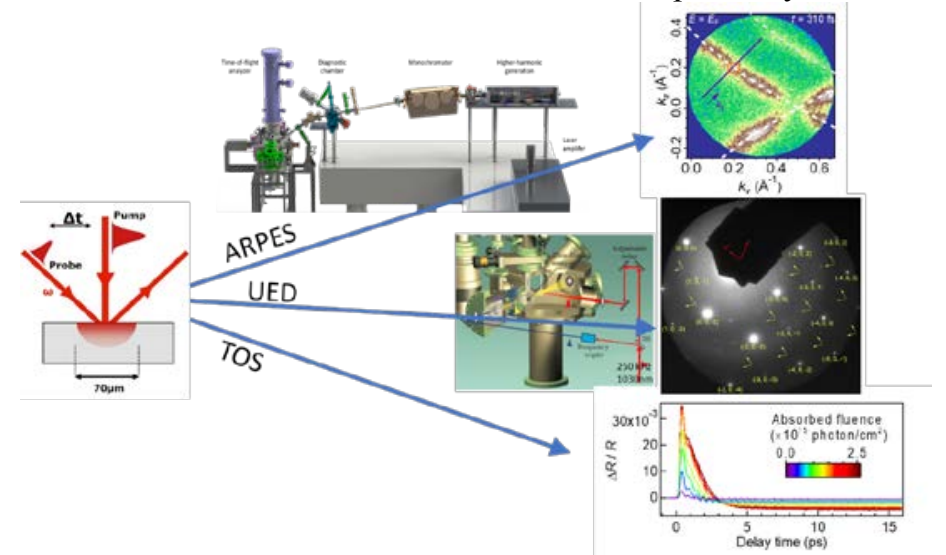

Fig. 1. The three different time-resolved techniques: trARPES, Ultrafast Electron Diffraction and Transient Optical Reflectivity. The left shows a typical pump probe setting while the center images present the experimental setup of the first two techniques. The right demonstrates data as acquired by the different detectors. The time-of flight detector acquires 3D data and only a cut near EF is plotted here. The superstructure peaks in UED are indicated by the yellow arrows.

For UED the same $\mathrm{Yb}: \mathrm{KGW}$ laser was employed. The fundamental wavelength at 1038 $\mathrm{nm}$ was used for excitation. The full $250 \mathrm{kHz}$ was used at low pump fluences of a few $\mu \mathrm{J} / \mathrm{cm}^{2}$. A pulse picker allowed us to use high pump fluences up to a few $\mathrm{mJ} / \mathrm{cm} 2$ at reduced repetition rates. For the probe pulses the fourth harmonic was generated and send to the photo-cathode in back-illumination. An ultracompact UED chamber with the focusing solenoid sitting on top of the electron gun was designed, enabling a propagation length of about an inch from the cathode to the sample to reduce space charge broadening. The e-beam energy was $26 \mathrm{keV}$ with a time-resolution of a few hundred fs. 
The air-sensitive samples were prepared by exfoliation down to less than $100 \mathrm{~nm}$ in a high-purity Argon glovebox for transmission electron diffraction.

TOS was performed with a Ti:Sa CPA laser system by using the fundamental light for excitation. The probe pulses were produced by white-light generation and the samples were kept in a cryogenic vacuum chamber. The differential reflection was acquired by a photodiode with a lock-in amplifier.

\section{Results}

$\mathrm{LaTe}_{3}$ is the most stable of the $\mathrm{RTe}_{3} \mathrm{CDW}$ compounds with an estimated transition temperature of $670 \mathrm{~K}$. In this material, the electronic system shows a fast recovery of the CDW superstructure gap on the timescale of a few hundred fs in good agreement with data on other $\mathrm{RTe}_{3}$ compounds [1, 6-7]. However, the superstructure peaks of the CDW as observed by UED have a strong fluence dependence on the recovery timescale and intensity, indicating a loss of the long range order of the superstructure. The recovery timescale in TOS is faster than in UED and in good agreement with the recovery observed by trARPES on the electronic system. However, the amplitude mode (AM), cp. oscillation in right bottom of Fig.1, depicts a fluence dependent quenching comparable to the lattice dynamics observed by UED when scaling the intensity of the AM by the peak excitation intensity. Moreover, the amplitude mode does not have a significant damping as would be expected for a breakdown of the phonon mode. This leads to the conclusion that the superstructure still exists locally, though it loses its long-range-coherence in the structural response due to the creation of topological defects, causing phase slips in the CDW.

In summary, the capability of combining modern time-resolved techniques has been shown by acquiring the dynamics over the full electron momentum dependence as well as the structural response and amplitude mode. Based on the progress to higher repetition rates, it was possible to reach a remarkable sensitivity and energy resolution.

A combined analysis of all three techniques paves the way to the interpretation that the amplitude mode is still alive, while the coherent phase of the CDW mode is quenched.

\section{References}

1. F. Schmitt, P. S. Kirchmann, U. Bovensiepen, R. G. Moore, L. Rettig, M. Krenz, J.-H. Chu, N. Ru, L. Perfetti, D. H. Lu, M. Wolf, I. R. Fisher, Z.-X. Shen, Science, 321, 1649 (2008)

2. L. X. Yang, G. Rohde, T. Rohwer, A. Stange, K. Hanff, C. Sohrt, L. Rettig, R. Cortés, F. Chen, D. L. Feng, T. Wolf, B. Kamble, I. Eremin, T. Popmintchev, M. M. Murnane, H. C. Kapteyn, L. Kipp, J. Fink, M. Bauer, U. Bovensiepen, and K. Rossnagel, Phys. Rev. Lett., 112, 207001 (2014)

3. Y. H. Wang, H. Steinberg, P. Jarillo-Herrero, and N. Gedik, Science 342, 453 (2013)

4. T. Rohwer, S. Hellmann, M. Wiesenmayer, C. Sohrt, A. Stange, B. Slomski, A. Carr, Y. Liu, L. Avila, M. Kalläne, S. Mathias, L. Kipp, K. Rossnagel, M. Bauer, Nature 471, 490 (2011)

5. L. Poletto and P. Villoresi, Appl. Opt. 45, 8577, (2006)

6. F. Schmitt, P. S. Kirchmann, U. Bovensiepen, R. G. Moore, J-H. Chu, D. H. Lu, L. Rettig, M. Wolf, I. R. Fisher, Z-X Shen, New J. Phys. 13, 063022 (2011)

7. L. Rettig, R. Cortes, J.-H. Chu, I.R. Fisher F. Schmitt, R.G. Moore, Z.-X. Shen, P.S. Kirchmann, M. Wolf, U. Bovensiepen, Nature Comm. 7, 10459 (2016) 